\title{
Concurrent Chemo-Radiotherapy for Locally Advanced Non-Small Cell Lung Cancer Using Intensity Modulated Radiotherapy Technique
}

\author{
Mohammed Tarrad ${ }^{1^{*}}$ MSc, Wael EL-Sheshtawy ${ }^{1}$ MD, Khaled Halima ${ }^{2}$ MD and Ahmed \\ El-Agamawy ${ }^{1} \mathrm{MD}$
}

\author{
Corresponding Author: \\ Mohammed Tarrad \\ dr.mohammed.tarrad.onc@gmail.com
}

Received for publication, May21, 2020; Accepted, July13, 2020; published online, July13, 2020.

\begin{abstract}
Copyright 2020 The Authors published by Al-Azhar University, Faculty of Medicine, Cairo, Egypt. All rights reserved. This an openaccess article distributed under the legal terms, where it is permissible to download and share the work provided it is properly cited. The work cannot be changed in anyway or used commercially.
\end{abstract}

doi:10.21608/aimj.2020.30559.1230

${ }^{1}$ Clinical Oncology \& Nuclear Medicine Department, Faculty of Medicine, Al-Azhar University, Cairo, Egypt

${ }^{2}$ Chest department, Faculty of medicine, Al-Azhar University

\begin{abstract}
Background: major progress in the management of non-small cell lung cancer emerged in the last years. Survival rate markedly improved in locally advanced non-small cell lung cancer with the use of concurrent chemo-radiotherapy treatment. Novel radiation techniques decreased the incidence of toxicity from radiotherapy.
\end{abstract}

Aim of the work: Assess toxicity, local control, progression-free and overall survival resulting from usage of concurrent chemo-radiotherapy treatment in locally advanced non-small cell lung cancer using intensitymodulated radiotherapy technique.

Patient and methods: this study included patients with stage III nonsmall cell lung cancer, received concurrent chemo-radiotherapy using intensity-modulated radiotherapy technique.

Results: out of 32 patients included $17(53.13 \%)$ were presented in stage IIIA and $15(46.87 \%)$ in stage IIIB. The commonest histo-pathological type was non-squamous cell carcinoma, it was detected in 21 patients $(65.62 \%)$, and squamous cell carcinoma which was detected in 11 patients (34.37\%). Partial response was achieved in $18(56.25 \%)$ patients, $10(31.25 \%)$ patients had stable disease and $4(12.5 \%)$ patients had progressive disease. Median overall survival was 10.2 months, while median progression-free survival was 6.5 months, no patients developed $\geq$ grade III esophagitis or pneumonitis.

Conclusion: Concurrent chemo-radiotherapy treatment with Paclitaxel Carboplatin, and intensity-modulated radiotherapy technique is a welltolerated treatment regimen with acceptable toxicity profile and fair survival in patients with un-resectable Stage IIIA-IIIB non-small cell lung cancer

Key words: Concurrent chemo-radiotherapy; advanced lung cancer; toxicity; survival.

Disclosure: The authors have no financial interest to declare in relation to the content of this article. The Article Processing Charge was paid for by the authors.

Authorship: All authors have a substantial contribution to the article. 
between $15 \%$ and $25 \%$, however, the survival rate of $30 \%$ was detected in some centers with trimodal treatment which includes surgical management. ${ }^{7,8}$

On the other hand, many platinum-based chemotherapy regimens have been given concurrently with RT including cisplatin/etoposide, cisplatin/vinblastine, carboplatin/pemetrexed, and cisplatin/pemetrexed. ${ }^{5,9,10}$

Right now, no consensus regarding which chemotherapy regimen is best to be given with radiotherapy. Although no standard definite chemotherapy regimen has been established for this approach, the low dose weekly paclitaxel and carboplatin regimen is preferred by many centers worldwide due to the acceptable toxicity profile and relative efficacy. ${ }^{9,11}$

It is worth noting that the management of locally advanced NSCLC is changing rapidly, now there is a role for consolidation immunotherapy after concurrent chemoradiotherapy in patients with partial response to treatment regardless of the patient's Programmed Death-Ligand (PD-L1) biomarker status and did not have EGFR mutations. ${ }^{12}$

This prospective study has been launched to evaluate the use of IMRT technique with concurrent weekly paclitaxel and carboplatin in patients presented with stage III NSCLC treated at our center.

\section{PATIENT AND METHODS}

This is a phase II (single-center experience) study included patients with locally advanced NSCLC, treated in the Department of Clinical Oncology and Nuclear Medicine at Al-Hussein University Hospital during the period between December 2016 and December 2019 .

All patients were having the following inclusion criteria; pathologically confirmed NSCLC, stage III disease, younger than 65 years old, and performance status (0-2) WHO at presentation.

Patients were excluded from the study if severe COPD (chronic obstructive pulmonary disease), severe cardiac problem, renal failure, patients with inadequate pulmonary functions tests [forced vital capacity (FVC) $>40 \%$ of the determined value, and forced expired volume at one second (FEV1) less than $1.5 \mathrm{~L}$, or $<60 \%$ of the determined value], prior surgery, radiotherapy or chemotherapy was given before, had a severe serious infection, or had a history of another malignancy.

Pretreatment evaluation included complete laboratory assessment, respiratory functions, chest, abdomen, and pelvis computed tomography (CT), bone scan, and brain magnetic resonance imaging (MRI).

After written informed consent, patients were planned to receive radiation therapy dose of $60 \mathrm{~Gy}$ in conventional fractionation (2 Gy per fraction, 5 days per week, over 30 fractions) using IMRT technique.

Treatment volumes were delineated as following, the gross tumor volume (GTV) which includes the primary tumor and positive regional lymph nodes, then a margin was used to generate the clinical target volume (CTV) (6 $\mathrm{mm}$ for squamous cell carcinoma and $8 \mathrm{~mm}$ for non-squamous histology). To compensate for respiratory motion, an internal target volume (ITV) has been used and finally, a 7 $\mathrm{mm}$ margin was added to CTV to generate a uniform planning target volume (PTV). Arc modified

Inverse planning IMRT was initiated for dose optimization using Varian software (ECLIPS version 13.6), consider using between 5-8 fields for this purpose. Verification was done by the portal image in the first 3 days, then weekly.

Patients were required to receive concurrent weekly Paclitaxel $45 \mathrm{mg} / \mathrm{m} 2$ and carboplatin [area under curve (AUC) 2] starting during the first 3 days of radiotherapy before receiving the session of radiotherapy treatment. Patients were seen regularly every week for an assessment during treatment, essential treatment modifications were done based on this assessment.

CT chest with contrast after 6 weeks from the end of c CRT was requested to assess the primary response. The assessment of response was done according to response evaluation criteria in solid tumors (RECIST) version 1.1.13 Further patients follow up was done using clinical examination and CT chest, abdomen and pelvis every 3 months during the first two years, then every 6 months to the end of year 5 , and yearly thereafter.

\section{Statistical methods}

Statistical package SPSS version 23 was used to code and enter data. Survival interval calculated as the time between the date of histological diagnosis and the date of last follow up (for censored observations) or date of death (for uncensored observations), while progression-free interval was examined as the time between the date of the first treatment and the date of the last follow-up (for censored observations) or; date of death or disease progression whatever happened first (for uncensored observations). Statistical analysis of overall survival and progression-free survival were done by one-sided log-rank of Kaplan-Meier survival estimates, while for univariate analysis of variables unpaired T-test and one-way ANOVA test were used. The results of the P-value are significant if > 0.05 .

\section{ETHICAL APPROVAL}

This study had been approved by the ethical board, Faculty of Medicine, Al-Azhar University, before the start of the study.

\section{RESULTS}

The median age of the included 32 patients was 60 (Range: 39-65) years, most of them were males 31 $(96.87 \%)$, with only one female patient $(3.12 \%)$. 
One patient $(3.12 \%)$ was non-smoker, 29 patients $(90.62 \%)$ were smokers and two patients $(6.25 \%)$ were EX-smokers.

The commonest histopathological type was, the non-squamous cell carcinoma, in 21 patients (65.62\%), while the squamous cell carcinoma, was detected in 11 patients $(34.37 \%)$. Only 6 patients (18.75\%) were evaluated by baseline Positron Emission Tomography-Computed Tomography (PET-CT) at presentation. The mean time to histological diagnosis from the first symptom was $5.5( \pm 3.75)$ months.

Almost all patients presented in performance status (PS) I, 30 patients $(93.75 \%)$, except 2 patients $(6.25 \%)$ presented in performance status II. seventeen patients $(53.13 \%)$ had stage IIIA and 15 patients $(46.87 \%$ ) had stage IIIB (Table 1 ).

The median number of IMRT fields were 6 (5-8) fields. The PTV coverage was between $95 \%$ and $107 \%$ and organs at risk (OAR) constraints has been achieved for all patients, with a median V20 Gy for both lungs-PTV of $20 \%$ (Range; $5 \%-35 \%$ ), V5 was $62 \%(18 \%-86 \%), \mathrm{V} 10$ was $41 \%(17 \%-63 \%)$, and the median mean lung dose was 12.25 (6.4-22.5) Gy. As regard heart, median heart V40 was 7\% (0\%-29\%), while for V45 was 5\% (0\%-25\%), and the median mean heart dose was 12 (1.4-26) Gy. The median mean esophageal dose was 24.25 (8.539) Gy, while the median esophageal Dmax was 61 (18-63) Gy. As regard the median Dmax of the spinal cord, it was 40.25 (8-48.6) Gy (Table 2).

\section{Toxicity pattern}

Patients (6.25\%) developed GI-II pneumonitis, and 3 patients $(9.38 \%)$ developed GIII pneumonitis. Regarding the hematological complications; 15 patients $(46.88 \%)$ developed G1-II anemia. As regard neutropenia, 24 patients $(74.99 \%)$ developed GI-II neutropenia, and two patients (6.25\%) developed GIII neutropenia. GIT complications, developed in the form of GI-II esophagitis in 15 patients $(46.87 \%)$. As regard to neurological complications, only $10(31.25 \%)$ patients developed GI neuropathy (Table 3).

The mean overall treatment days was $43.46( \pm 7.30)$ (20-57) days, and the maximum delay was 15 days. The delay occurred due to different causes mainly anemia which causes a delay for 5 patients (15.62\%), GII esophagitis which presented in 4 patients $(12.5 \%)$, and chest infection in another 4 patients $(12.5 \%)$. Another cause of delay was an irregularity on treatment and this presented in 2 patients $(6.25 \%)$ (Table 3$)$.

Response to treatment was evaluated at six weeks from the end of treatment, the partial response was the most frequent one, as it presented in more than half of patients (18 from 32) $(56.25 \%$ ), the next was disease progression, as it presented in (10 from 32) $(31.25 \%)$ of patients, finally, the stationary disease presented in 4 patients from $32(12.5 \%)$, One of the patients in the progression category died during the treatment course due to pneumonia, patient performance status was II, stage IIIB, her histopathology was undifferentiated carcinoma, duration of symptoms before diagnosis was 9 months, received only 17 fractions of her planned radiotherapy treatment, the patient developed type II respiratory failure followed by death.

\section{Survival analysis}

After a median follow up of 6 months, the median progression-free survival (PFS) time was 6.5 months and median overall survival (OS) time was 10.2 months (Figure 1, 2). 


\begin{tabular}{|c|c|c|}
\hline Factors & & Total $\mathbf{n}=\mathbf{3 2}$ \\
\hline \multirow[t]{4}{*}{ Age } & mean & SD \\
\hline & 57.41 & 8.37 \\
\hline & median & Range \\
\hline & 60 & $39-65$ \\
\hline$\underline{\text { Sex }}$ & $\mathbf{N}$ & $\%$ \\
\hline Female & 1 & 3.12 \\
\hline Male & 31 & 96.87 \\
\hline$\underline{\text { Smoking status }}$ & $\mathbf{N}$ & $\%$ \\
\hline Non-smoker & 1 & 3.12 \\
\hline Smoker & 29 & 90.62 \\
\hline EX-smoker & 2 & 6.25 \\
\hline Histopathology & $\mathbf{N}$ & $\%$ \\
\hline Non-squamous cell & 21 & 65.62 \\
\hline Squamous cell & 11 & 34.37 \\
\hline Baseline PET-CT & 6 & 18.75 \\
\hline \multirow{4}{*}{$\begin{array}{c}\text { Duration of symptoms before diagnosis } \\
\text { (month) }\end{array}$} & Mean & SD \\
\hline & 5.53 & 3.75 \\
\hline & Median & Range \\
\hline & 1 & $(5-12)$ \\
\hline$\underline{\mathrm{PS}(\mathrm{WHO})}$ & $\underline{\mathbf{N}}$ & $\underline{\%}$ \\
\hline I & $\overline{30}$ & $9 \overline{3.75}$ \\
\hline II & 2 & 6.25 \\
\hline$\underline{\text { T stage }}$ & $\underline{\mathbf{N}}$ & $\underline{\%}$ \\
\hline 1 & $\overline{1}$ & 3.12 \\
\hline 2 & 4 & 12.5 \\
\hline 3 & 12 & 37.5 \\
\hline 4 & 15 & 46.88 \\
\hline N stage & $\underline{\mathbf{N}}$ & $\underline{\%}$ \\
\hline No & 6 & 18.75 \\
\hline N1 & 8 & 25 \\
\hline $\mathbf{N 2}$ & 15 & 46.88 \\
\hline N3 & 3 & 9.38 \\
\hline Final stage & $\underline{\mathbf{N}}$ & $\underline{\%}$ \\
\hline IIIA & $\overline{17}$ & 53.13 \\
\hline IIIB & 15 & 46.87 \\
\hline
\end{tabular}

Table 1: Demographic and Clinical Characteristics

\begin{tabular}{|c|c|c|c|c|c|}
\hline Factors & Mean & SD & Median & Min & Max \\
\hline PTV Dose V95\% & $99 \%$ & $1 \%$ & $99 \%$ & $95 \%$ & $100 \%$ \\
\hline Lungs minus PTV (V20) & $20 \%$ & $8 \%$ & $20 \%$ & $5 \%$ & $35 \%$ \\
\hline V5 & $62 \%$ & $15 \%$ & $62 \%$ & $18 \%$ & $86 \%$ \\
\hline V10 & $40 \%$ & $11 \%$ & $41 \%$ & $17 \%$ & $63 \%$ \\
\hline MLD (GY) & 12.3 & 3 & 12.25 & 6.4 & 22.5 \\
\hline heart V40 & $9 \%$ & $8 \%$ & $7 \%$ & $0 \%$ & $29 \%$ \\
\hline heartV45 & $7 \%$ & $7 \%$ & $5 \%$ & $0 \%$ & $25 \%$ \\
\hline heart MHD(GY) & 12.37 & 7.39 & 12 & 1.4 & 26 \\
\hline Esophageal Mean (GY) & 24.34 & 7.17 & 24.25 & 8.5 & 39 \\
\hline Esophageal Dmax (GY) & 56.88 & 10.95 & 61 & 18 & 63 \\
\hline Spinal cord Dmax (GY) & 36.85 & 10.84 & 40.25 & 8 & 48.60 \\
\hline & & & 6 & 5 & 8 \\
\hline
\end{tabular}

Table 2: Dose of coverage and constraints 


\begin{tabular}{|c|c|c|}
\hline \multirow[t]{2}{*}{ Factors } & \multicolumn{2}{|c|}{ Total $\mathbf{n}=32$} \\
\hline & $\mathbf{N}$ & $\%$ \\
\hline \multicolumn{3}{|l|}{ Pneumonitis } \\
\hline GI-II & 2 & 6.25 \\
\hline GIII & 3 & 9.38 \\
\hline \multicolumn{3}{|l|}{ Anemia } \\
\hline GI-II & 15 & 46.88 \\
\hline \multicolumn{3}{|l|}{ Neutropenia } \\
\hline GI-II & 24 & 74.99 \\
\hline GIII & 2 & 6.25 \\
\hline \multicolumn{3}{|l|}{ Esophagitis } \\
\hline GI-II & 15 & 46.87 \\
\hline \multicolumn{3}{|l|}{ Neuropathy } \\
\hline GI & 10 & 31.25 \\
\hline \multirow[t]{2}{*}{ Overall treatment days } & $\frac{\text { Mean }}{43.46}$ & $\frac{\text { SD }}{7.30}$ \\
\hline & $\frac{\text { Median }}{42}$ & $\frac{\text { Range }}{(20-57)}$ \\
\hline \multirow[t]{2}{*}{ Days of delay } & $\frac{\text { Mean }}{3.21}$ & $\frac{\text { SD }}{5.25}$ \\
\hline & $\frac{\text { Median }}{0}$ & $\frac{\text { Range }}{(0-15)}$ \\
\hline Cause of delay & No & $\%$ \\
\hline No & 17 & 53.12 \\
\hline GII esophagitis & 4 & 12.5 \\
\hline Chest infection & 4 & 12.5 \\
\hline Patient was irregular & 2 & 6.25 \\
\hline Anemia & 5 & 15.62 \\
\hline
\end{tabular}

Table 3: Toxicity pattern and duration of treatment with radiotherapy

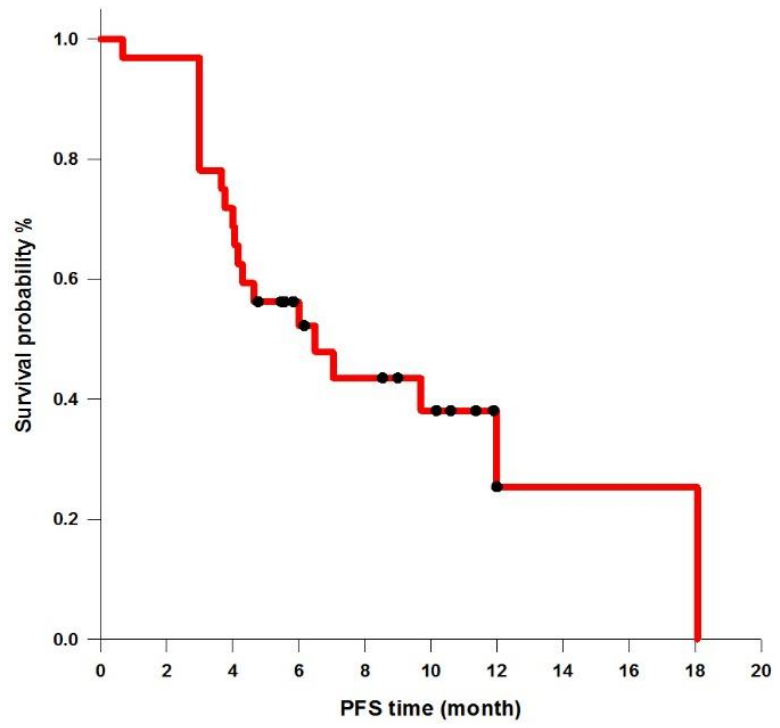

Fig. 1: Kaplan Meier survival curve for PFS time 


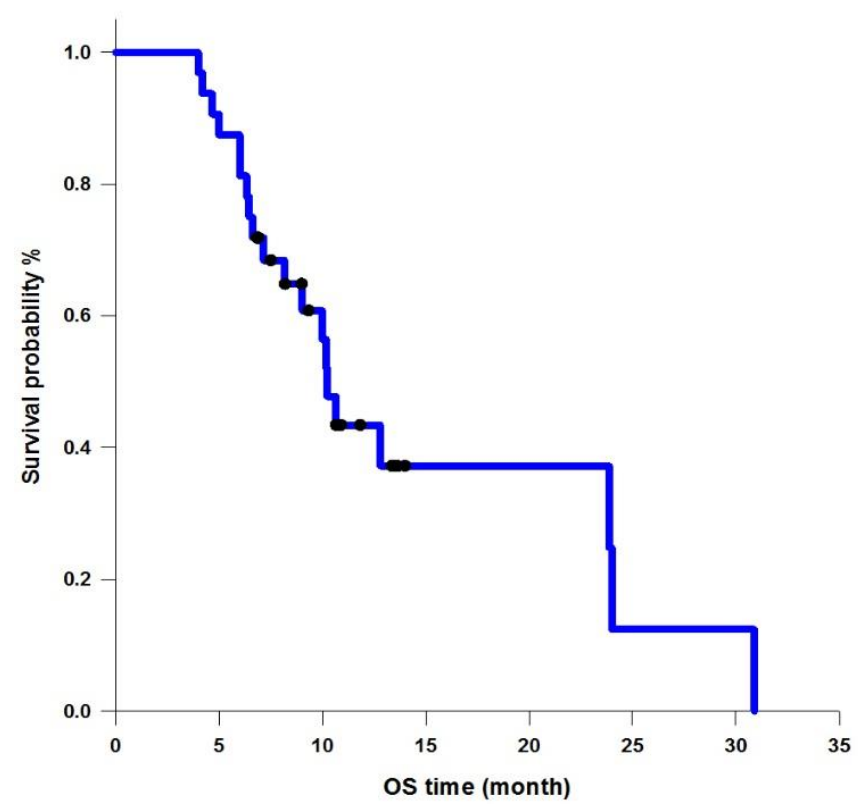

Fig. 2: Kaplan Meier survival curve for OS time

\section{DISCUSSION}

One-third of NSCLC patients presented in a locally advanced stage. Inoperable stage III NSCLC represent $70 \%$ of NSCLC patients, therefore, lung cancer (LC) remains an important challenge for oncology care today. ${ }^{14}$

The number of patients included in our study was small, although more than one hundred patients presented to our center during the period of recruitment, most of the patients were stage IV disease or with stage III disease but with a poor functional status, or has other comorbid conditions that disqualified them from enrollment in our study.

Weekly paclitaxel and carboplatin is a common regimen to be used concurrently with radiotherapy, but there is currently no consensus regarding which chemotherapy regimen is best to combine with radiotherapy. ${ }^{9}$

The chemotherapy regimen used in the Radiation Therapy Oncology Group (RTOG) 0617 trial was carboplatin/paclitaxel in the same doses used in our study with good tolerability, the study presented only $\geq$ grade III toxicity, which was in minimal presentation. ${ }^{11}$

Our study demonstrated satisfactory tolerability to weekly paclitaxel/carboplatin, as only a very small number of patients developed grade III neutropenia, with no anemia $\geq$ grade II in any patient, neuropathy was not also frequent except in diabetic patients and was not more than grade II.

On the other hand; the role of radiotherapy in the treatment of locally advanced NSCLC is essential especially intensity-modulated radiotherapy technique (IMRT) which offers an advantage over conventional three-dimensional radiotherapy (3DRT) in these settings. ${ }^{15}$

The key clinical question is whether IMRT technique in combination with chemotherapy can lead to an improvement in OS as a result of reduced toxicity rate? In our study, we have studied the role of IMRT in improving dose coverage, managing large tumor volume and its role in decreasing lung toxicity, and its effect on response rate and survival . The PTV coverage in our study was optimal. As regard Dmax, and mean dose of the esophagus with IMRT was perfect. On the other hand, the heart V40, V45Gy and mean doses were within the acceptable dose constraints in all patients, furthermore maximum dose delivered to the spinal cord was within tolerance even in tumors with close proximity to vertebrae.

Lung V20 dosimetric constraint is considered a threshold dose that can determine the chance of lung toxicity. ${ }^{16}$

In our study, meaningful low lung V20 Gy and mean lung dose was detected.

John and his colleges discussed the dosimetric advantage of the IMRT technique in locally advanced NSCLC, the results demonstrated the superiority of IMRT regarding normal tissue exposure. In terms of lung dose, statistically significant reductions in mean lung dose and V20 Gy was obtained without affecting lung V5 Gy. As regard esophageal dosimetric constraints, IMRT showed a perfect reduction in esophageal mean dose, V20 Gy, V45 Gy, and V60 Gy. Finally, for heart dosimetric constraints, IMRT improved V5 Gy, V30 Gy and V45 Gy. ${ }^{17}$

A correlation was done between radiation pneumonitis (RP) and low-dose baths, as lung V5 in a retrospective analysis. In patients with stage III NSCLC treated by IMRT technique, low dose baths were not a predictor for radiation pneumonitis, the two most commonly-utilized parameters as a predictor for radiation pneumonitis are the normal bilateral lung volume received $\geq 20 \mathrm{~Gy}$ (minus the planning target volume) and the mean lung dose (MLD). ${ }^{18}$ 
We have evaluated V5 and V10 in our study, the median V5 was $62 \%(18-86 \%)$ and V10 was $41 \%$ (17-63\%) within tolerance. Although lung V5 Gy was higher in patients treated with IMRT, it was not accompanied by $\geq$ grade III toxicity .

In RTOG 0617 trial, values of lung V5 were high, IMRT provided preferable lung toxicity than 3DCRT. This study suggested that lung V5 is not a predictor of toxicity of radiotherapy treatment in locally advanced NSCLC. ${ }^{16}$

As regard, acute toxicity related to radiotherapy treatment using IMRT technique. In RTOG 0617, IMRT was not accompanied with pneumonitis or esophagitis $\geq$ grade III, only $(5 \%)$ of patients experienced $\geq$ grade 3 esophagitis, and $20.6 \%$ of patients experienced pulmonary toxicity, and on a multivariable analysis of RTOG 0617 study, a statistically significant decrease in pneumonitis risk was achieved with IMRT technique. ${ }^{16}$ In our study, a small number of patients developed pneumonitis grade III $(9.38 \%)$, no patient developed grade IV pneumonitis. Also, esophagitis was in the form of GI and II only, although chemotherapy exerts independent and potentially synergistic effects with thoracic radiation therapy on the esophagus .

RTOG 91-06, 92-04, and 94-10 trials were analyzed to determine if overall treatment period can affect survival in patients with locally advanced NSCLC treated with concurrent chemoradiotherapy, severe acute esophagitis was associated with long treatment period, patients finished concurrent chemoradiotherapy treatment on time showed better median overall survival (19.5 months vs. 14.8 months). ${ }^{19}$

Unfortunately, in our study there was an interruption during the concurrent chemoradiotherapy treatment period in 15 patients, the mean delay time was 3.21 (0-15) days, this delay was due to various causes like anemia, esophagitis, chest infection and irregularity of the patients .

RTOG 9410 study demonstrated marked improvement in survival and response rate with the concurrent chemoradiotherapy arms. Median survival time (MST) was 17 months and 15.6 months in arm 2 and 3 respectively which used concurrent chemoradiotherapy treatment, versus 14.6 in the sequential arm. ${ }^{5}$ The previous findings were also assessed in phase I-II trial of concurrent chemoradiotherapy treatment with weekly paclitaxel and carboplatin in locally advanced non-small-cell lung cancer, the phase II part of the study revealed RR of 93.8\%; PFS of 11.7 months, and OS of 24 months. ${ }^{20}$

In our study, the response rate was evaluated using RECIST criteria, partial response was seen in $(56.25 \%)$ patients, while the progressive and stationary disease was seen in $(31.25 \%)$ and $(12.5 \%)$ patients respectively, which is not far from the reported results by RTOG 9410 .

In our study, median progression-free survival was 6.5 months, and the median overall survival was 10.2 months. Poor survival outcome in our study may be explained by the relatively large number of cases presented in T4 and stage IIIB disease due to the late diagnosis, lower number of patients $6(18.75 \%)$ that staged using PET-CT scan before the start of treatment, although now PET scan is standard in staging workup of lung cancer because it has a major role in providing information about metabolic activity of the tumor, mediastinal involvement and detecting occult metastases. ${ }^{21}$

Other factors affected survival outcome in our study were prolonged treatment time due to interruption of the course of treatment in some patients, the bad nutritional status, lower socioeconomic level of included patients, and unavailability of recent drugs as immunotherapy that could be used as a consolidation after concurrent chemoradiotherapy treatment.

\section{CONCLUSION}

Concurrent chemoradiotherapy using IMRT technique is a well-tolerated regimen in selected inoperable Stage III NSCLC patients with a good response rate and fair survival. However further larger studies are required to look for more active systemic regimens and advanced radiation techniques for this category of patients.

\section{REFERENCES}

1. Ferlay J, Colombet M, Soerjomataram I, et al. Estimating the global cancer incidence and mortality in 2018: GLOBOCAN sources and methods. Int J Cancer. 2019 Apr 15;144(8):19411953.

2. Ibrahim AS, Khaled HM, Mikhail NNH, et al. Cancer incidence in Egypt: results of the National Population-Based Cancer Registry Program. J Cancer Epidemiol. 2014; 437971.

3. Fournel P, Robinet G, Thomas P, et al. Randomized phase III trial of sequential chemoradiotherapy compared with concurrent chemoradiotherapy in locally advanced nonsmall-cell lung cancer: Groupe Lyon-SaintEtienne d'Oncologie Thoracique-Groupe Français de Pneumo-Cancérologie NPC 95-01 Study. $J$ Clin Oncol. 2005 Sep 1;23(25):5910-7. Epub 2005 Aug 8.

4. Aupérin A, Le Péchoux C, Pignon JP, et al. Concomitant radio-chemotherapy based on platin compounds in patients with locally advanced non-small cell lung cancer: A meta-analysis of individual data from 1764 patients Ann Oncol 17: 473-483,2006.

5. Curran WJ Jr, Paulus R, Langer CJ, et al. Sequential vs. concurrent chemo-radiation for stage III non-small cell lung cancer: randomized phase III trial RTOG 9410. J Natl Cancer Inst 2011; 103(19):1452-60.

6. Kilburn JM, Soike MH, Lucas JT, et al. Image guided radiation therapy may result in improved local control in locally advanced lung cancer patients. Pract Radiat Oncol 2016 May-Jun;6(3): e73-e80.

7. Pless M, Stupp R, Ris H-B, et al. Induction chemoradiation in stage IIIA/N2 non-small-cell lung cancer: a phase 3 randomised trial. Lancet. 2015 Sep 12;386(9998):1049-56.

8. Eberhardt WE, Pöttgen C, Gauler TC, et al. Phase III Study of Surgery Versus Definitive 
Concurrent Chemoradiotherapy Boost in Patients With Resectable Stage IIIA(N2) and Selected IIIB Non-Small-Cell Lung Cancer After Induction Chemotherapy and Concurrent Chemoradiotherapy (ESPATUE). J Clin Oncol. 2015;33 (35): 4194-4201. doi:10.1200/JCO.2015.62.6812

9. Steuer CE, Behera M, Ernani V, et al. Comparison of concurrent use of Thoracic Radiation with Either Carboplatin- Paclitaxel or Cisplatin-Etoposide for Patients with Stage III Non-Small-Cell Lung Cancer: A Systematic Review. JAMA Oncol. 2017 Aug 1;3(8):11201129 .

10. Senan S, Brade A, Wang LH, et al. PROCLAIM: randomized phase III trial of pemetrexedcisplatin or etoposide-cisplatin plus thoracic radiation therapy followed by consolidation chemotherapy in locally advanced nonsquamous non-small-cell lung cancer. J Clin Oncol. 2016 Mar 20;34(9):953-62.

11. Bradley JD, Paulus R, Komaki R, et al. Standarddose versus high dose conformal radiotherapy with concurrent and consolidation carboplatin plus Paclitaxel(e instead of i) with or without Cetuximab for patients with stage IIIA or IIIB non-small cell lung cancer (RTOG 0617): a randomized, two by two factorial phase III study. Lancet Oncol. 2015 Feb; 16(2): 187-99.

12. Antonia SJ, Villegas A, Daniel D, et al. Overall survival with durvalumab after chemoradiotherapy in stage III NSCLC. N Engl J Med. 2018 Dec 13;379(24):2342-2350.

13. Schwartz LH, Litière $\mathrm{S}$, de Vries $\mathrm{E}$, et al. RECIST 1.1 - Update and Clarification: From the RECIST Committee. Eur J Cancer. 2016 Jul;62:132-7.

14. Malvezzi M, Carioli G, Bertuccio P, et al. European cancer mortality predictions for the year 2017, with focus on lung cancer. Ann Oncol. 2017 May 1;28(5):1117-1123.

15. Tejan P, Pranshu Mohindra, Melissa Vyfhuis, et al. Advances in radiotherapy techniques and delivery for non-small cell lung cancer: benefits of intensity- modulated radiation therapy, proton therapy, and stereotactic body radiation therapy. Transl Lung Cancer Res: 2017 Apr; 6(2): 131147.

16. Chun SG, Hu C, Choy $\mathrm{H}$, et al. Impact of Intensity-Modulated Radiation Therapy Technique for Locally Advanced Non-SmallCell Lung Cancer: A Secondary Analysis of the NRG Oncology RTOG 0617 Clinical Trial. J Clin Oncol. 2017; 35 (1):56-62. Epub 2016 Oct 31.

17. John Boyle, Brad Ackerson, Lin Gu, et al. Dosimetric advantages of intensity modulated radiation therapy in locally advanced lung cancer. Adv Radiat Oncol.2017 Jan-Mar; 2(1): 6-11 .

18. Meng Y, Yang H, Wang W, et al. Excluding PTV from lung volume may better predict radiationpneumonitis for intensity modulated radiation therapy in lung cancer patients. Radiation Oncology (London, England), 14 Jan 2019, 14(1):7.

19. Machtay M, Hsu C, Komaki R, et al. Effect of overall treatment time on outcomes after concurrent chemoradiation for locally advanced non-small-cell lung carcinoma: analysis of the Radiation Therapy Oncology Group (RTOG) experience. Int J Radiat Oncol Biol Phys.2005 Nov 1;63(3):667-71. Epub 2005 May 31.

20. Takamatsu K, Marumo S, Takagi T, et al. Phase I/II study of weekly paclitaxel and carboplatin with concurrent radiation therapy in locally advanced non-small-cell lung cancer: Kansai Clinical Oncology Group T0401. Integr Mol Med (2017), 4: DOI: 10.15761/IMM.1000300.

21. Sharma P, Singh H, Basu S, Kumar R, et al. Positron emission tomography-computed tomography in the management of lung cancer. An update South Asian J cancer. 2013;2(3):171178 .

22. Ford CN, Reinhard ER, Yeh D, et al. Interim analysis of a prospective, randomized trial of vacuum-assisted closure versus the healthpoint system in the management of pressure ulcers. Ann Plast Surg. 2002;49 (1):55-61. doi:10.1097/00000637-200207000-00009

23. Chen S-Z, Li J, Li X-Y, Xu L-S. Effects of vacuum-assisted closure on wound microcirculation: an experimental study. Asian $J$ Surg. 2005;28 (3):211-217. doi:10.1016/S10159584(09)60346-8

24. Philbeck TE, Whittington KT, Millsap MH, Briones RB, Wight DG, Schroeder WJ. The clinical and cost effectiveness of externally applied negative pressure wound therapy in the treatment of wounds in home healthcare Medicare patients. Ostomy Wound Management. 1999; 45(11):41-50.

25. Bickels J, Kollender Y, Wittig JC, Cohen N, Meller I, Malawer MM. Vacuum-assisted wound closure after resection of musculoskeletal tumors. Clin Orthop Relat Res. 2005;441:346-350. doi:10.1097/01.blo.0000180450.21350.3e

26. Armstrong DG, Lavery LA, Boulton AJM. Negative pressure wound therapy via vacuumassisted closure following partial foot amputation: what is the role of wound chronicity? Int Wound J. 2007;4(1):79-86. doi:10.1111/j.1742-481X.2006.00270. 